\title{
A First-Principle Study of Monolayer Transition Metal Carbon Trichalcogenides
}

\author{
Muhammad Yar Khan ${ }^{1} \cdot$ Yan Liu $^{1} \cdot$ Tao Wang $^{1} \cdot$ Hu Long ${ }^{1} \cdot$ Miaogen Chen $^{2} \cdot$ Dawei Gao $^{1}$
}

Received: 2 June 2021 / Accepted: 16 July 2021 / Published online: 27 July 2021

(c) The Author(s) 2021

\begin{abstract}
Monolayer $\mathrm{MnCX}_{3}$ metal-carbon trichalcogenides have been investigated by using the first-principle calculations. The compounds show half-metallic ferromagnetic characters. Our results reveal that their electronic and magnetic properties can be altered by applying uniaxial or biaxial strain. By tuning the strength of the external strain, the electronic bandgap and magnetic ordering of the compounds change and result in a phase transition from the half-metallic to the semiconducting phase. Furthermore, the vibrational and thermodynamic stability of the two-dimensional structure has been verified by calculating the phonon dispersion and molecular dynamics. Our study paves guidance for the potential applications of these two mono-layers in the future for spintronics and straintronics devices.
\end{abstract}

Keywords Metal-carbon trichalcogenides $\cdot$ Density functional theory $\cdot$ Electron density of states $\cdot$ Magnetic properties

\section{Introduction}

Two-dimensional materials have shown high potential for the realization of nano-devices in the near future. Reducing the thickness of materials down to a mono (or few) atomic layer has drawn enormous interest because they could reveal unusual trends that are distinct from their bulk counterparts, e.g., Quantum spin Hall effect, 2D ferroelectricity, magnetism, and superconductivity [1-6] Motivated by the incredible improvement of graphene, other 2D materials have been a noticeable research field of concern. Graphene, the first successfully exfoliated essential material in 2D limit with the honeycomb lattice, possesses linear band dispersion crossing at the K-point in the 1st Brillouin zone (BZ),

Tao Wang

twang@zju.edu.cn

$\triangle$ Dawei Gao

dawei_gao@163.com

1 School of Materials Science \& Engineering, School of Micro-Nano Electronics, Second Affiliated Hospital Zhejiang University College of Medicine, Zhejiang University, Hangzhou 310027, People's Republic of China

2 Key Laboratory of Intelligent Manufacturing Quality Big Data Tracing and Analysis of Zhejiang Province, Department of Physics, China Jiliang University, Hangzhou 310018, People's Republic of China generating the Dirac Fermions. The two-dimensional dichalcogenide materials have attracted concentration for the last few decades due to their various application such as spintronics, electronic sensors, memory devices, and field-effect transistors [7, 8]. Many stimulating 2D materials like hexagonal boron nitride $(h-\mathrm{BN})$ and dichalcogenides have been studied experimentally by various researchers [9-12].

Various computational studies have been presented to examine the nature of two-dimensional dichalcogenides, the chromium-containing ternary tritellurides $\mathrm{CrSiTe}_{3}$ and $\mathrm{CrGeTe}_{3}, \mathrm{MnP} X_{3}$ ternary chalcogenides, and $\mathrm{CrX}_{3}$ trihalides. The vanadium-based dichalcogenides $\mathrm{V} X_{2}(X=\mathrm{S}, \mathrm{Se})$ were first recommended and reported for their strain-tunable ferromagnetic phase [13]. The chromium-trihalides $\mathrm{Cr} X_{3}$ $(X=\mathrm{F}, \mathrm{Cl}, \mathrm{Br}, \mathrm{I})$ [13-19] are a new class of semiconducting ferromagnetic materials with a high Curie temperature $\left(\mathrm{T}_{\mathrm{C}}<100 \mathrm{~K}\right)$. Attempts have been made to transform $2 \mathrm{D}$ materials into magnetic by straining, doping, or finding inherently magnetic 2D materials. Previous research was focused mainly on doping the non-magnetic 2D materials along with transition magnetic metals, e.g., Co functionalized graphene [20].

Density functional theory (DFT) studies have grouped TM trichalcogenides with the general formula $\mathrm{MnCX}_{3}$ $(\mathrm{X}=\mathrm{Se} \mathrm{Te})$. Bulk compounds in this family have already been known [19, 21-23], but recently mono- and few layers of these compounds have been successfully exfoliated 
$[16,18,20,24-34]$. One major lead of the $\mathrm{MnCX}_{3}$ family is that it has a very wide range of chemical diversity as well as structural complexity. Compounds in the $\mathrm{MnCX}_{3}$ family display interesting properties such as half-metallicity or semiconducting coupled with ferromagnetic (FM) and antiferromagnetic (AFM) order [7, 8, 16, 18-20, 23-35].

Keeping in view the aforementioned theoretical and experimental extraordinary methods, the biaxial strain is the main parameter for the phase transition. Excited from these methods here in this study, we emphasize the electronic and magnetic properties of monolayer $\mathrm{MnA} X_{3}$, as well as the mutual coupling amongst the strain and magnetism, which are thoroughly examined using density-functional theory (DFT). The transition from FM to AFM is observed by applying strain on these monolayers. To confirm the AFM stability after applying biaxial as well as uniaxial strain, we calculated various configurations of AFM, i.e., Neel antiferromagnetic (nAFM), zigzag antiferromagnetic (zAFM), striped antiferromagnetic (sAFM), and ferromagnetic (FM). We investigate the electronic structures improve when the magnetic phases change from ferromagnetic to antiferromagnetic. Our results show a robust mutuality between magnetism and structural properties in $\mathrm{MnCX}_{3}$ materials, owing to an amazingly strong reliance on exchange interaction forces on the strain. Our calculations demonstrate that the race between antiferromagnetic and ferromagnetic order can be significantly increased by strain engineering since these materials include transition metals and thus have strong dependence. The goal of this study is to provide an understanding into expected materials-property trends and to study the viability of engineering magnetic properties in these materials employing biaxial as well as uniaxial strain.

\section{Computational Methods}

All the calculations are carried out by density functional theory (DFT) executed in the Vienna Ab-initio Simulation Package (VASP) [36]. The generalized gradient approximation (GGA) with the parameterization scheme of Perdew-Burke-Ernzerhof (PBE) is applied for the electron exchange-correlation processes, while the electron-ion interactions are processed by the projector augmented wave (PAW) method [37]. Hubbard model is applied to comprise the contribution of the on-site strong interactions of the localized $d$ electrons of the $\mathrm{Mn}$ $(U=4 \mathrm{eV})$ atoms. The cutoff energy of $500 \mathrm{eV}$ is selected, for the plane-wave expansion of the electronic eigenfunctions, for the monolayer $\mathrm{MCX}_{3}$. The total energy of the structure and force criterion for the atomic relaxation are chosen as $10^{-5} \mathrm{eV}$ and 0.01 respectively, so that the structures fully become relaxed. It should be noticed that for the phonon calculations, we have considered EDIFF $=10^{-7} \mathrm{eV}$ in order to minimize the error to a maximum possible extent. The periodic images of monolayers of $\mathrm{MCX}_{3}$ are separated by $20 \AA$ to bypass the interlayer interactions $K$-point sampling with a $7 \times 7 \times 1$ mesh is used for the integration of the Brillouin zone (BZ). The displacement step size is selected as $0.015 \AA$ using four displacements for each ion in the $\mathrm{x}$ and $\mathrm{y}$ directions. Phonon calculations are performed using the finite displacement method as implemented in the phonopy program [38].

\section{Result and Discussion}

\subsection{Structural Properties}

We predict two new members of the $\mathrm{MnCX}_{3}$ family $\mathrm{MnCTe}_{3}$ and $\mathrm{MnCSe}_{3}$ monolayers, whose bulk structures have been discovered in past experiments [16, 20,39-41]. The space group of these two recently predicted structures is P-31 M. Therefore, the addition of these two members will expand and broaden the scope of this family. The unit cell of $\mathrm{MnCTe}_{3}$ and $\mathrm{MnCSe}_{3}$ is shown with green color in Fig. 1.

The two Mn atoms in the unit cell have sixfold coordination with $\mathrm{Se} / \mathrm{Te}$ atoms, creating octahedra, whereas the carbon atoms have threefold coordination with the $\mathrm{Se} / \mathrm{Te}$ atoms. The relaxed lattice parameters for $\mathrm{MnCTe}_{3}$ and $\mathrm{MnCSe}_{3}$ monolayers are noted as $a=b=6.633 \AA$, $a=b=6.126 \AA$, and $\alpha=120^{\circ}$. The lattice parameters and structure thickness are linked to the dimension of the chalcogen atoms, since the length of $\mathrm{C}-\mathrm{C}$ dimers expands. Moreover, the bond lengths in $\mathrm{MnCTe}_{3}$ regarding $\mathrm{Mn}-\mathrm{Te}$ and $\mathrm{Mn}-\mathrm{Mn}$ are $2.86 \AA 3.83 \AA$ respectively, whereas the $\mathrm{Mn}-\mathrm{Te}-\mathrm{Mn}$ bond angle is $84.04^{\circ}$. The Mn-Se bond length $(2.65 \AA)$ in $\mathrm{MnCSe}_{3}$ is shorter due to the smaller radius of $\mathrm{Se}$ compared to Te atom. Due to the

a)

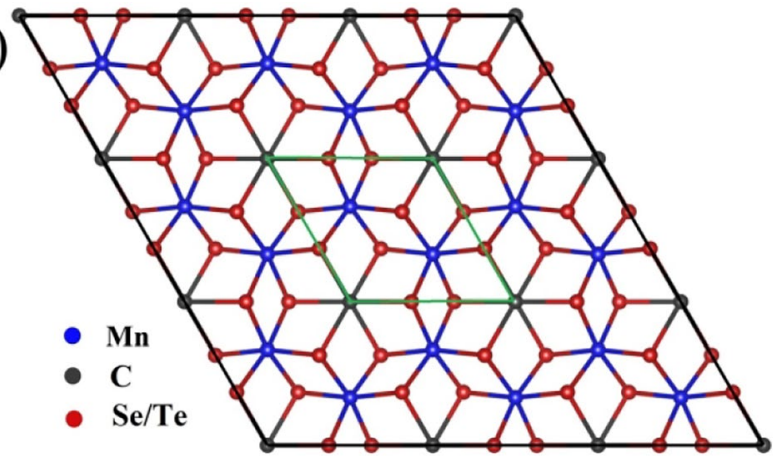

b)

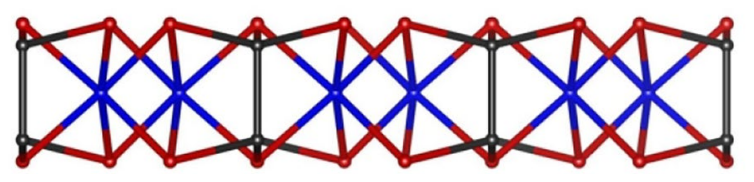

Fig. 1 Top a and side $\mathbf{b}$ views of crystal structure of $\operatorname{MnCX}_{3}(X=\mathrm{Xe}$, $\mathrm{Se})$ compounds. Green box represents the unit cell, where two transition metal atoms assemble in a honeycomb structure 
smaller Se atomic size, the $\mathrm{C}-\mathrm{C}$ dimer size is also reduced. As a result, the $\mathrm{Mn}-\mathrm{Mn}$ bond length (3.53 $\AA$ ) gets shorten. However, the $\mathrm{Mn}-\mathrm{Se}-\mathrm{Mn}$ bond angle is slightly reduced.

\subsection{Magnetic Properties}

We explore the ground-state (GS) magnetic ordering of the $\mathrm{MnCX}_{3}(\mathrm{X}=\mathrm{Te}, \mathrm{Se})$ by comparing their GS energies. The GS of the $\mathrm{MnCTe}_{3}$ and $\mathrm{MnCSe}_{3}$ are $-49.68 \mathrm{eV}$ and $-54.19 \mathrm{eV}$ for FM while $-48.95 \mathrm{eV}$ and $-51.47 \mathrm{eV}$ for AFM case respectively. We also calculate different configurations of AFM, i.e., Neel (nAFM) $-97.903 \mathrm{eV}$, zigzag AFM (zAFM), $-97.900 \mathrm{eV}$ and stripy AFM (sAFM) $-98.6437 \mathrm{eV}$, and ferromagnetic (FM) $-98.6949 \mathrm{eV}$, which show that FM state is more stable than the AFM. The difference between the ground-state energy of the antiferromagnetic (AFM) and ferromagnetic (FM) order $\left(E_{\mathrm{AFM}}-E_{\mathrm{FM}}\right)$ reveals that both systems energetically favor FM ordering. We tested different values for the on-site Coulomb interactions to evaluate the GS energies. After computing the GS energies, we realized that the band gap converge is at around $U=4 \mathrm{eV}$. Therefore, for the rest of the calculations, we adopt $\mathrm{U}=3 \mathrm{eV}$ for the strength of the on-site Coulomb interaction. The formal valences of the atoms are $\mathrm{Mn}^{2+}, \mathrm{C}^{4+}, \mathrm{Te}^{-2}$, and $\mathrm{Se}^{-2}$. According to Hund's rule, $\mathrm{Mn}^{2+}$ with a $3 \mathrm{~d}^{5}$ electronic configuration reveals a high spin state. The magnetic moments are developed largely at the localized $\mathrm{Mn}$ atom sites in the ferromagnetic configuration. In the FM state, every Mn atom provides $4.335 \mu \mathrm{B}$, whereas, in the AFM state, the two Mn atoms in the unit cell participate $\pm 4.219 \mu \mathrm{B}$. Magnetism requires two key parameters: (1) local magnetic moment, (2) spins-coupling. Here, Te and Se act as a mediator among $\mathrm{Mn}-\mathrm{Mn}$ coupling which leads to superexchange interaction.

Our results demonstrate that the ferromagnetism persists in the monolayer layer of $\mathrm{MnCX}_{3}(\mathrm{X}=\mathrm{Se}, \mathrm{Te})$. In a 2D $\mathrm{MnCX}_{3}$ crystal, the significantly larger bond-length between the Mn-Mn magnetic atoms omits the possibility of direct exchange. Therefore, the exchange interactions among the metal atoms are thus largely facilitated by indirect exchange interactions. The carbon atom weakly hybridizes with the transition-metal atoms as can be clearly seen from the PDOS in Fig. 2a, c. However, the d-orbitals of the Mn considerably hybridize with chalcogen atoms (Se, Te). The chalcogen atoms carry tiny negative magnetic moments and they couple antiferromagnetically with their surrounding $\mathrm{Mn}$ atoms. Thus, this indirect coupling encases the superexchange interaction.

\subsection{Electronic Properties}

To investigate the electronic properties of the $\mathrm{MnCTe}_{3}$ and $\mathrm{MnCSe}_{3}$ monolayers, we calculate the spin-polarized band structures and partial density of states (PDOS). The
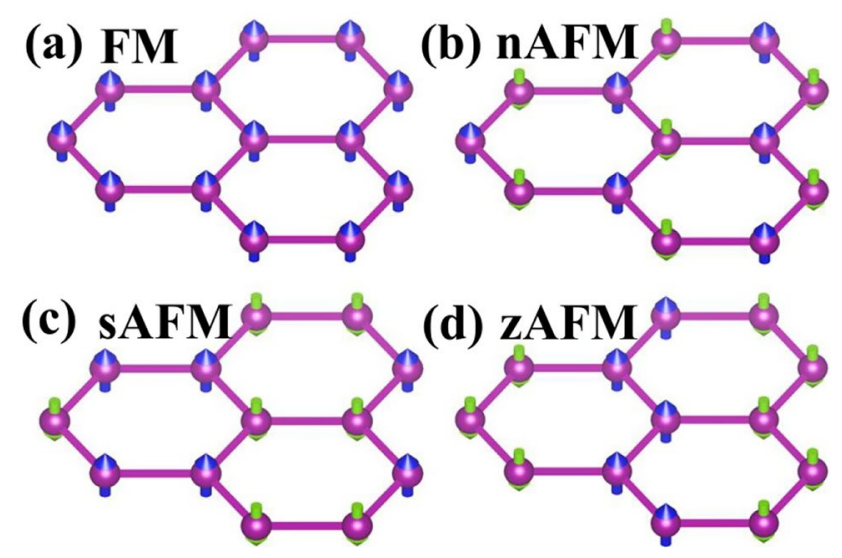

Fig. 2 Schematic interpretations of different magnetic ordering in $\mathrm{MnCX}_{3}$ compounds. a Ferromagnetic, b Néel antiferromagnetic, c stripy antiferromagnetic, and d zigzag antiferromagnetic

band structures as well as the PDOS of the $\mathrm{MnCTe}_{3}$ and $\mathrm{MnCSe}_{3}$ reveal that both the systems possess half-metallic behaviors in their ground states which can be clearly seen in Fig. 3. The spin-up channel conducts as it crosses the Fermi level, while the spin-down does not conduct. The spin-down channels $\mathrm{MnCTe}_{3}$ and $\mathrm{MnCSe}_{3}$ remain insulating with finite gaps $(1.78 \mathrm{eV}$ and $1.049 \mathrm{eV})$ respectively. In addition to $M n-t_{2 g}$ states, the band structures of the former and latter are fully spin-polarized at the Fermi level by the Te-p and Se-p orbitals respectively. Moreover, PDOS depicts that both systems possess a large spin splitting gap which makes the half metallicity robust against external perturbation. The large spin-splitting gaps (1.78 eV for $\mathrm{MnCSe}_{3}$ and $1.049 \mathrm{eV}$ for $\mathrm{MnCTe}_{3}$ ) make them promising materials for spintronic applications since they can provide a hundred percent spin-polarized carriers. This gap between spin-up and spin-down channels is located around the fermi level. Thus, $2 \mathrm{D} \mathrm{MCX}_{3}$ compounds gain half-metallic character due to exchange splitting around the fermi level. Importantly, the spin-splitting gap can be notably enhanced or suppressed by applying biaxial or uniaxial strain. Interesting features of the band structures are the presence of the Dirac points which is not observed in other transition metal trichalcogenides.

\subsection{Phonon Dispersions and Molecular Dynamics Simulations}

After proving structural stability, we performed phonon dispersion calculations, see Fig. 4. Phonon spectra of the $\mathrm{MnCX}_{3}$ monolayers are computed to validate the dynamic stability of $\mathrm{MnCSe}_{3}$ and $\mathrm{MnCTe}_{3}$ monolayers. The maximum frequency of $\mathrm{MnCTe}_{3}$ is smaller compared to that of the $\mathrm{MnCSe}_{3}$ monolayer because Te is heavier than Se. The lack of soft phonon modes in the first Brillion zones calculated through high symmetry points for the monolayer 
Fig. 3 (Color online) PDOS of monolayer a $\mathrm{MnCTe}_{3}$ and $\mathbf{c}$ $\mathrm{MnCSe}_{3}$. The corresponding contribution of the atoms is depicted with different colors. In the PDOS plots, the negative and positive parts represent the majority and minority spin channels. The Fermi level $\left(\mathrm{E}_{\mathrm{F}}\right)$ is fixed at zero. Band structure of $\mathbf{b} \mathrm{MnCTe}_{3}$ and $\mathbf{d} \mathrm{MnCSe}_{3}$. Moreover, the blue- and redcolored lines represent the bands of majority and minority spin bands
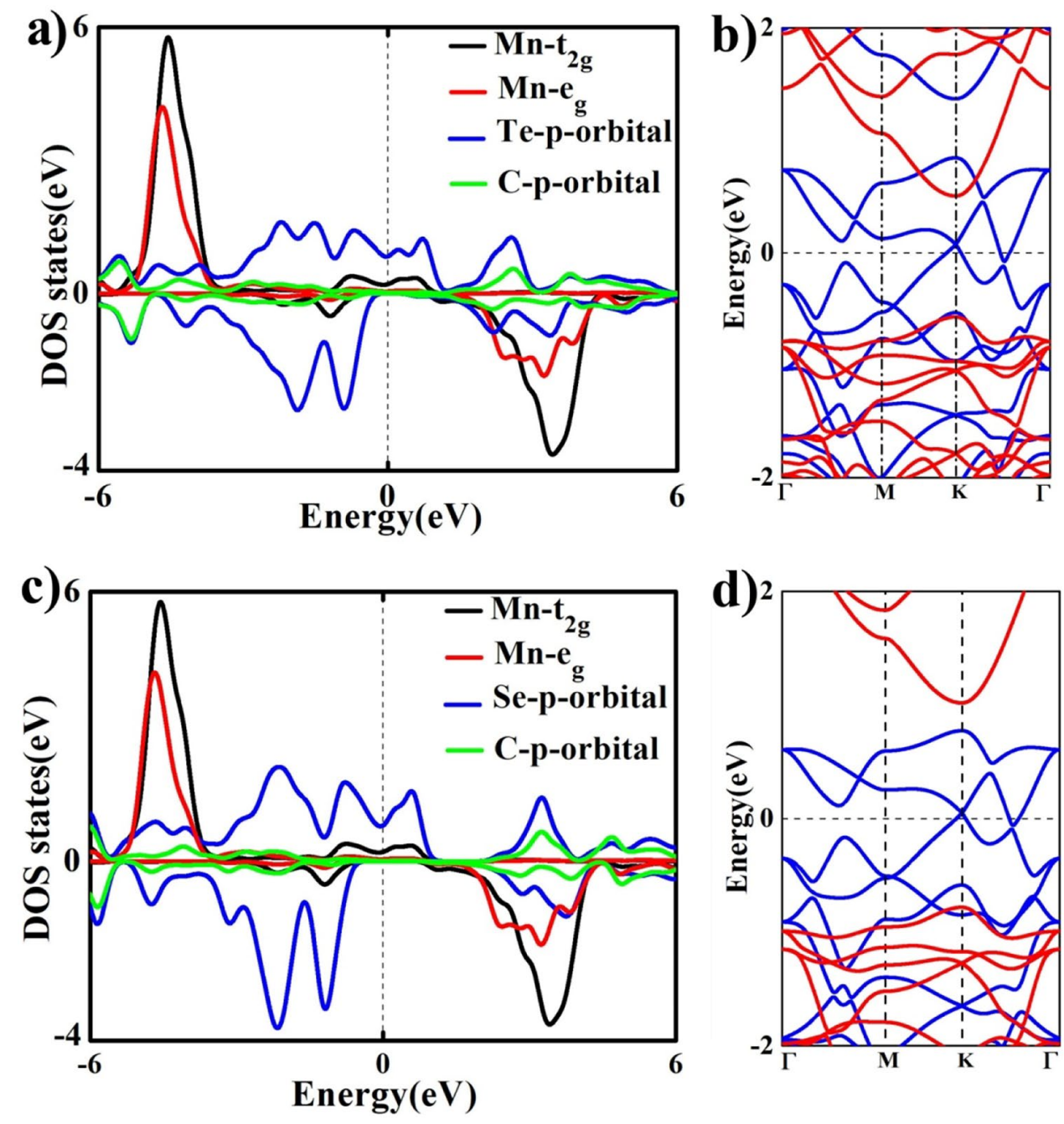
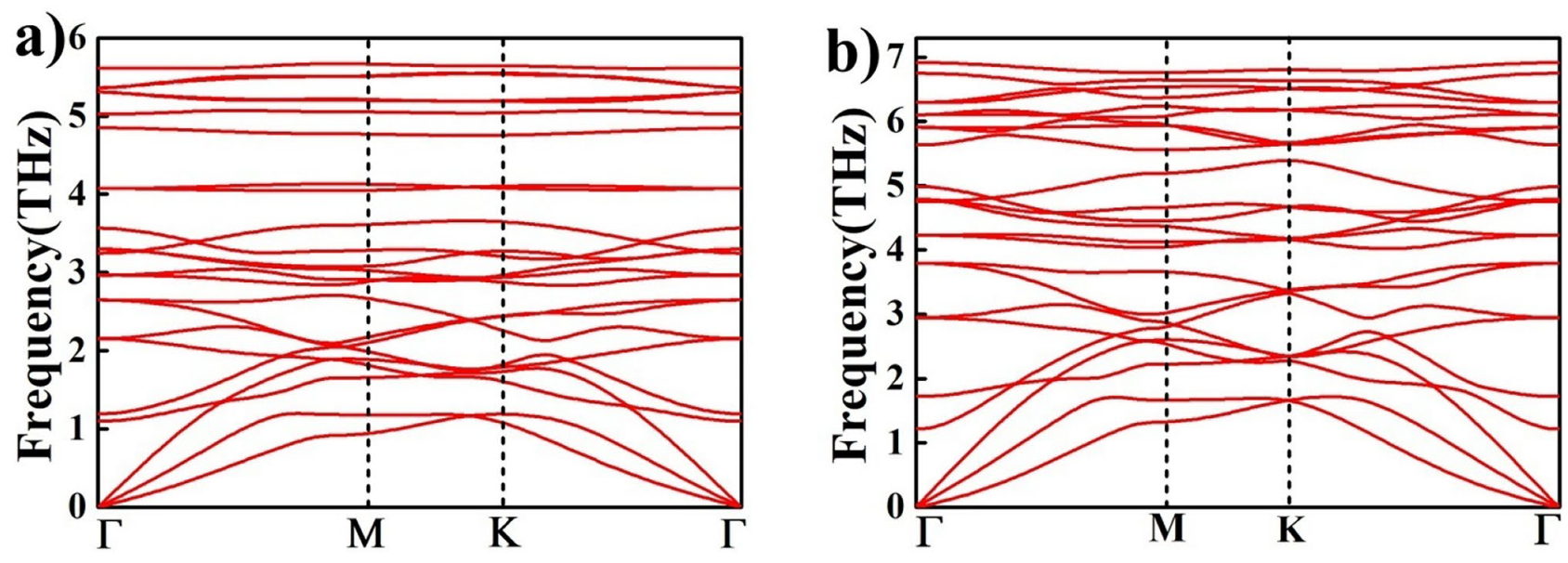

Fig. 4 Phonon band dispersions for monolayer $\mathbf{a ~} \mathrm{MnCTe}_{3}$ and $\mathbf{b} \mathrm{MnCSe}_{3}$, respectively 
$\mathrm{MnCSe}_{3}$ and $\mathrm{MnCTe}_{3}$ is the indication for their dynamic stability, in analogy to monolayer $\mathrm{W}_{2} \mathrm{C}$, WS, WSe, MoC, WC, and many others [42-45]. First, three modes with relatively smaller frequencies are the acoustic modes whereas the rest with higher frequencies are the optical modes. Each mode is because of specific lattice vibration. There are three branches of the acoustic modes, longitudinal (LA), transverse (TA), and out-of-plane (ZA). The LA and TA branches show linear while the ZA shows a quadratic behavior neat to the gamma point which is a characteristic of the free-standing single layers, claimed as a result of the point group symmetry [46, 47]. Our findings are consistent with the earlier reported phonon band dispersions for other stable monolayers [44-46].

We further study the thermodynamic stability of monolayers $\mathrm{MnCTe}_{3}$ and $\mathrm{MnCSe}_{3}$ at $300 \mathrm{~K}$ by performing the ab initio molecular dynamics simulations with a time step of $1 \mathrm{fs}$ for 7.5 ps (Fig. 5). We observed that the energy fluctuations with time are very small. Also, the atoms of the lattice vibrate from its lattice site and reconstruct the structure. It shows that both these structures are stable at room temperature.

\subsection{Strain-Induced Magnetic Phase Transition}

2D materials are very delicate due to their thin nature and therefore essentially show a decent response to external stimuli, like strain, pressure, and doping. Motivated by such feature, we subjected both the monolayers of $\mathrm{MnCTe}_{3}$ and $\mathrm{MnCSe}_{3}$ to external strains which may allow to tune and enhance their magnetic and electronic properties. To know the mechanism of the phase transitions caused by the strain, we examine the dependence of magnetic properties against strain variations. The local magnetic moment appears on $\mathrm{Mn}$ atoms in $\mathrm{MnCTe}_{3}$ and $\mathrm{MnCSe}_{3}$. At the ground state, the two $\mathrm{Mn}$ atoms in the unit

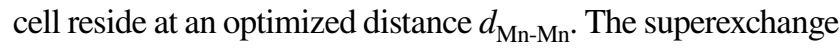
interaction tends to favor FM order between the local magnetic moments of the Mn atom. However, we explore that both the systems are quite sensitive to the external strain. The strain $\varepsilon$ can be defined as:

$\varepsilon=\frac{\left(a-a_{0}\right)}{a} \times 100$
Fig. $5 \mathrm{Ab}$ initio molecular dynamic simulations at $300 \mathrm{~K}$ for $7.5 \mathrm{pc}$ with a time step of 1 fs for $\mathbf{a} \mathrm{MnCTe}_{3}$ and $\mathbf{b}$ $\mathrm{MnCSe}_{3}$, respectively

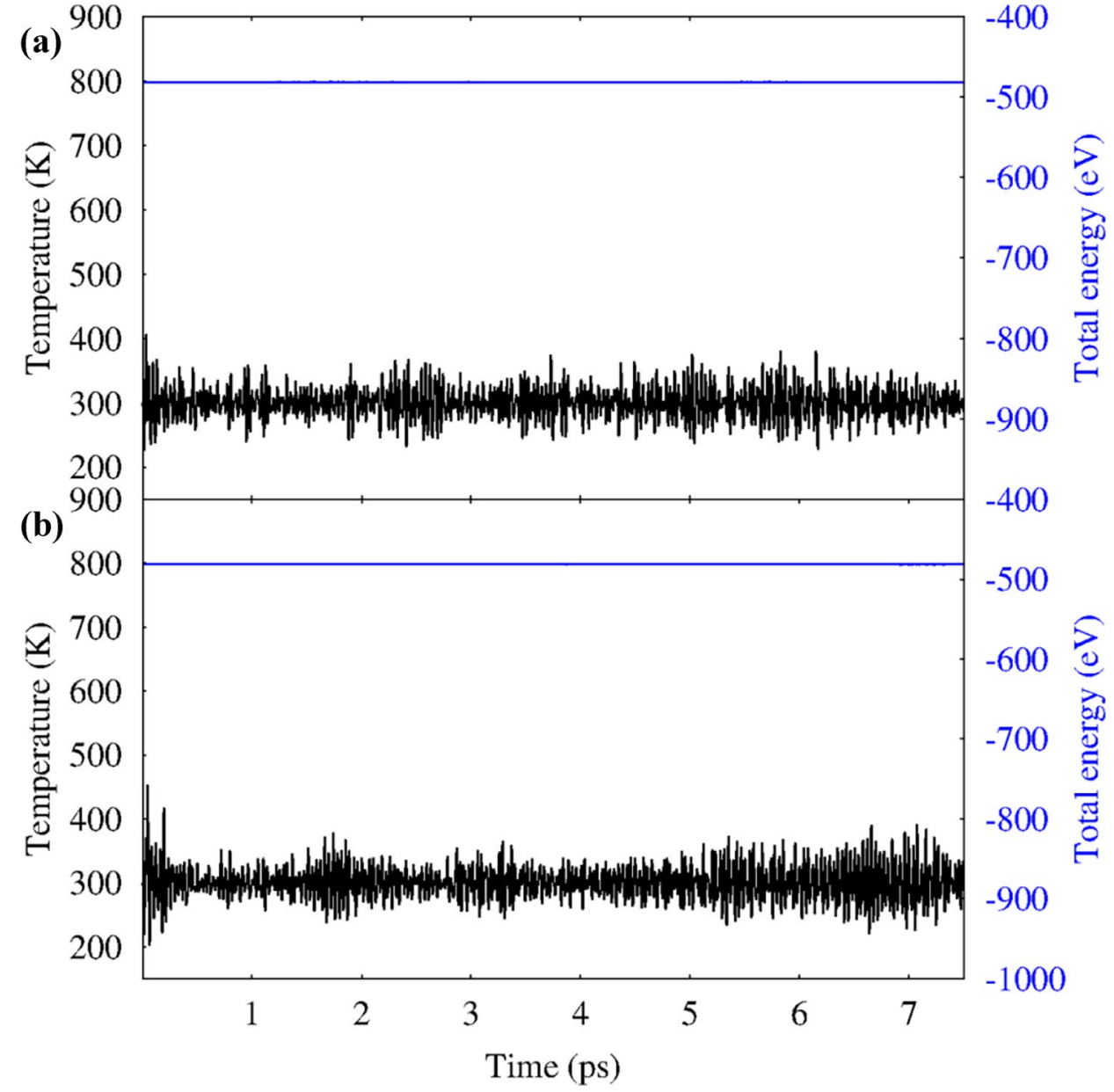


where $\mathrm{a}_{0}$ and $a$ are the lattice parameter of the system with and without strain respectively. We assume that the energy difference between FM and AFM by including the contribution of the second and the third nearest neighbors. Therefore, the exchange energy due to the contribution of the first nearest neighbor can be written as:

$E_{\mathrm{FM}}-E_{\mathrm{AFM}}=-6 J|\vec{S}|^{2}$

where $S$ is the spin and $J$ is the exchange parameter. If $E_{\mathrm{FM}}-E_{\mathrm{AFM}}$ is known, then the exchange parameter $J$ can be computed via Eq. (2) from the energy difference calculation. The Currie temperature with the available value of $J$ can be calculated by the mean-field expression:

$T_{\mathrm{c}}=\frac{3 J}{2 K_{B}}$

where $T_{c}$ and $K_{b}$ represent the Currie temperature and Boltzmann constant. Eq. 3 shows that the Curries temperature can be increased by increasing the exchange energy.

For a clearer picture, we plot the exchange energy $\left(\mathrm{E}_{\mathrm{AFM}}-\mathrm{E}_{\mathrm{FM}}\right)$ against externally applied strain in Fig. 6, which depicts that the FM states, regarding both the systems, are robust against tensile strain. Importantly, the stability of the ground state of the FM state increases with increasing tensile strain. However, its stability decreases with compressive strain and $\mathrm{E}_{\mathrm{AFM}}-\mathrm{E}_{\mathrm{FM}}$ switches its sign at some value, where magnetic phase transition takes place from FM to AFM order. The direct exchange interaction varies with the distance between two local moments as $\approx 1 / \mathrm{d}^{5}{ }_{\mathrm{Mn}-\mathrm{Mn}}[46]$ and it promotes antiferromagnetism in a system. With tensile strain, the two nearest magnetic $\mathrm{Mn}$ atoms move away from each other. As a result, the direct exchange reduces drastically whereas the superexchange also reduces but it is not affected significantly. The net effect enhances the FM order in both systems. By contrast, in compressive strain, the nearest magnetic atoms get closer and closer to each other. Therefore, a direct exchange is enhanced drastically while superexchange does not increase at that much pace. At some significant compressive strain, the direct exchange completely dominates and the systems go under magnetic phase transition from FM to stripy AFM (sAFM) state. In particular, the critical values of strain for $\mathrm{MnCTe}_{3}$ and $\mathrm{MnCSe}_{3}$ are $-3 \%$ and $-1 \%$ respectively. Our analysis shows that the superexchange dominates for tensile strain. For the compressive strain, direct exchange and superexchange complete each other; however, the direct exchange dominates beyond a particular value and brings phase transition in both systems. It is important to mention that a similar result was reported by Zheng et al. for $\mathrm{CrI}_{3}$ under compressive strain [48]. Moreover, the compressive strain also influences the electronic structure and alters the half-metallic state of $\mathrm{MnCTe}_{3}$ and $\mathrm{MnCSe}_{3}$ at the critical value of strain as shown in Fig. 7a-f as well as from half-metallic to semiconductor state. Additionally, by increasing the compressive strain, i.e., from -3 to $-7 \%$, the strain does not significantly change the bandgap. The strain effects on band gaps give a handy method to control the bandgap by design via epitaxy. For both these systems, strains $-3 \%,-5 \%$, and $-7 \%$; the VBM; and the CBM of the total density of states (TDOS) TDOS are mainly dominated by the Mn-d, Te-p, and Se-p orbitals. The contribution of the $\mathrm{C}$ atom is very small to DOS, so we neglect in the partial density of states. From Fig. 7, one can see a significant hybridization between the Te- $p$, Se- $p$, and Mn- $d$ states. We believe that $\mathrm{MnCTe}_{3}$ and $\mathrm{MnCSe}_{3}$ are promising materials for spintronic devices since they depict ferro- and antiferromagnetism and show many interesting electronic behaviors like half metallicity semiconducting state.
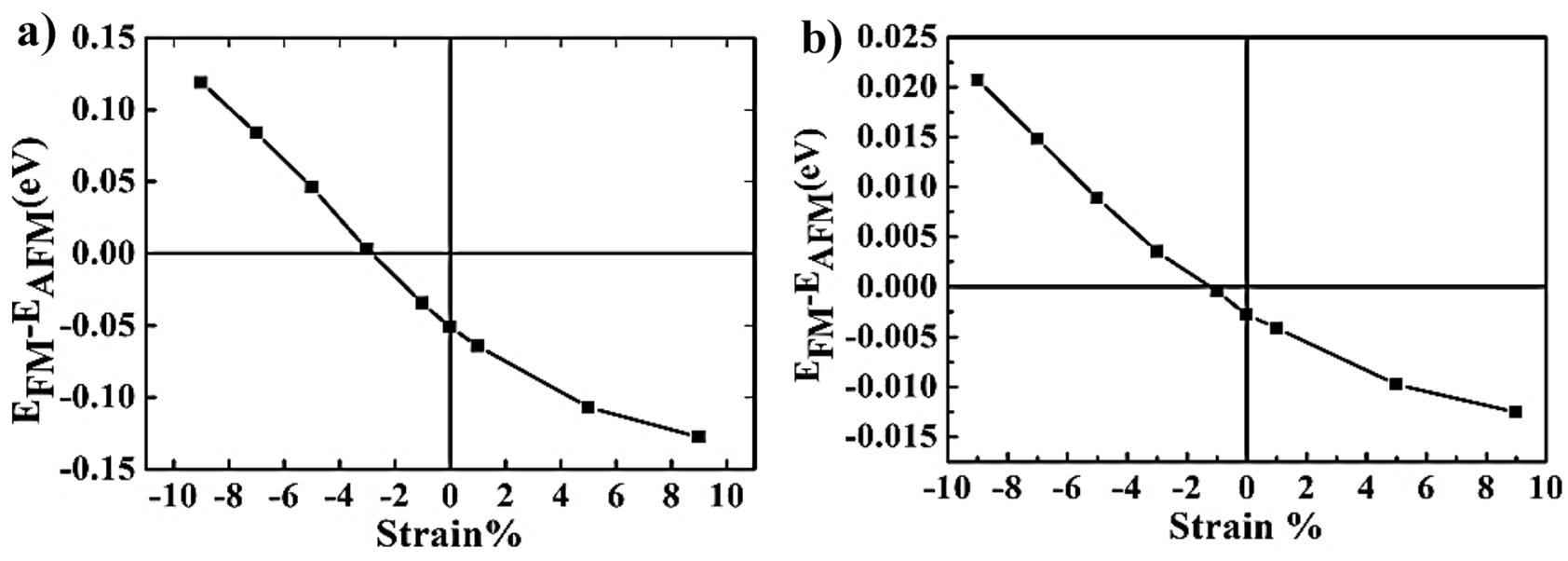

Fig. 6 Energy difference between FM and AFM phases for $\mathbf{a} \mathrm{MnCTe}_{3}$ and $\mathbf{b} \mathrm{MnCSe}_{3}$ 

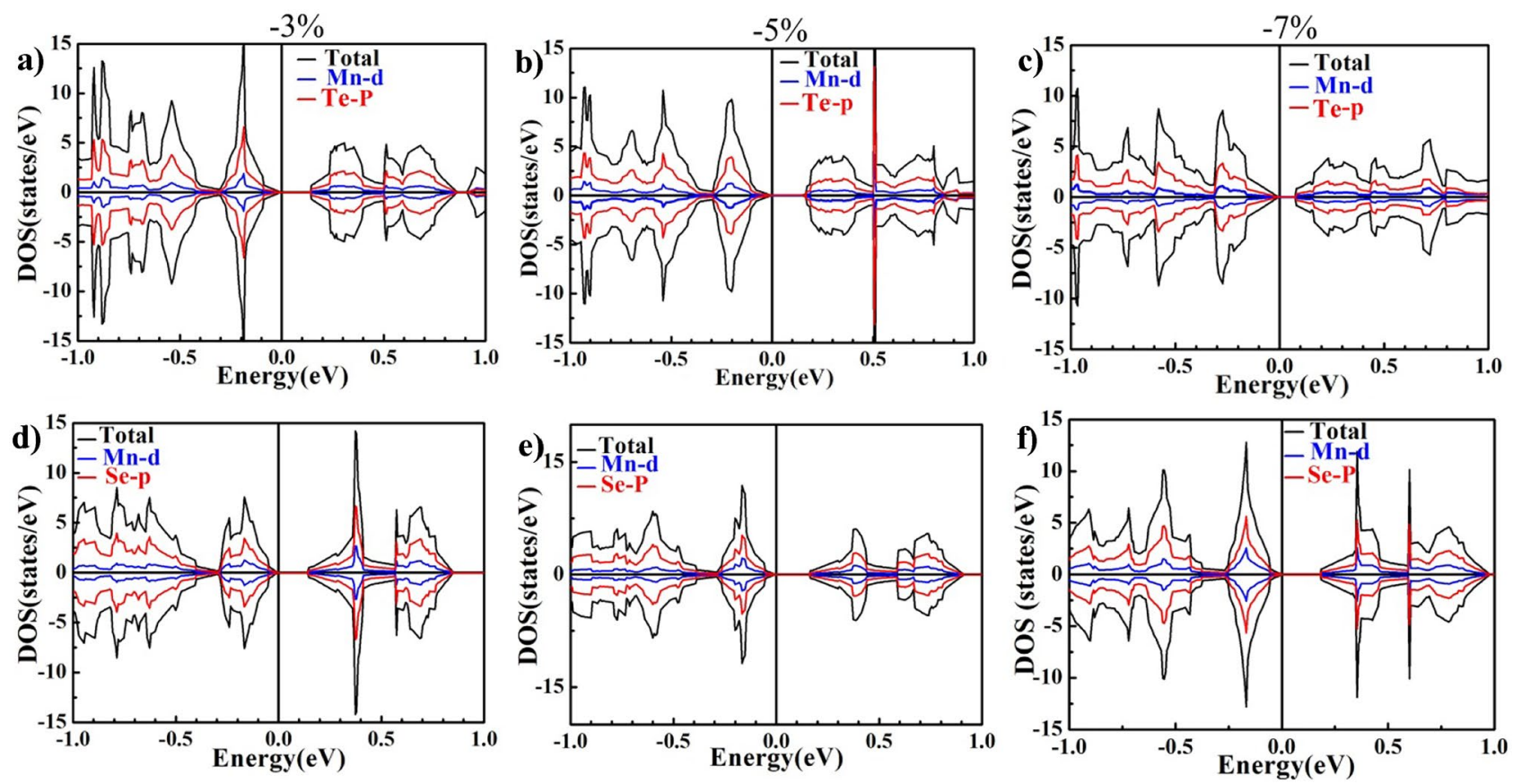

Fig. 7 TDOS and partial density of states of $\mathbf{a}-\mathbf{c} \mathrm{MnCTe}_{3}$ and $\mathbf{d}-\mathbf{f} \mathrm{MnCSe}_{3}$ with $-3 \%,-5 \%$, and - 7\% strains, respectively

\section{Conclusions}

In this paper, we accomplished comprehensive first-principle calculations in order to investigate the structural, magnetic, and electronic properties of the $\mathrm{MnCX}_{3}(\mathrm{X}=\mathrm{Se}, \mathrm{Te})$ to broaden the scope of this family for the realization of future spintronics applications. Firstly, we verified their dynamical stability by evaluating the phonon dispersions. We did not find a negative band in the phonon dispersion which clearly depicts their dynamic stability. We further confirmed their thermodynamic stability by performing the ab initio molecular dynamic simulations. The small fluctuations with time in the energies guarantee its thermal stability. Our results indicate that van der Waal 2D $\mathrm{MnCX}_{3}(\mathrm{X}=\mathrm{Se}, \mathrm{Te})$ favors the ferromagnetic phase in the monolayer limit. We notice that the magnetic moment hinge largely depends on the transition metal atoms. The band structures of both the member inherent half-metallic nature with respect to the majority spin channel. The PDOS depicts that the Mn- $d$ contributes around the Fermi level. Moreover, we have analyzed the impact of biaxial as well as uniaxial strain and the impact of strain on the magnetic and electronic properties. Our results indicate that the magnetic ordering can be manipulated by strain engineering because it substantially sets a competing environment between FM and AFM order. By analyzing the effects of the strain, we found that FM order gets more stable with tensile train for both species regarded $\mathrm{MnCX}_{3}$. However, the systems $\mathrm{MnCTe}_{3}$ and $\mathrm{MnCSe}_{3}$ undergo a magnetic phase transition (from FM to AFM order) upon applying compressive strain of $3 \%$ and $1 \%$ respectively. The AFM ordering is getting more and more stable with increasing compressive strain. The compressive strain greatly affects the electronic structures of the 2D monolayer $\mathrm{MnCTe}_{3}$ and $\mathrm{MnCSe}_{3}$. The half-metallic states transform into semiconducting at particular compressive strain. The intrinsic halfmetallic character and the sensitivity of the exchange coupling of these systems to external agents such as strain make them incredible room for new functionalities in magnetic 2D materials. Based on our calculations, we conclude that monolayers $\mathrm{MnCTe}_{3}$ and $\mathrm{MnCSe}_{3}$ are exciting candidate materials for $2 \mathrm{D}$ spintronics.

Funding This work was supported by the National Key R\&D Program of China (2019YFE0112000) and the Fundamental Research Funds for the Provincial Universities of Zhejiang.

Open Access This article is licensed under a Creative Commons Attribution 4.0 International License, which permits use, sharing, adaptation, distribution and reproduction in any medium or format, as long as you give appropriate credit to the original author(s) and the source, provide a link to the Creative Commons licence, and indicate if changes were made. The images or other third party material in this article are included in the article's Creative Commons licence, unless indicated otherwise in a credit line to the material. If material is not included in the article's Creative Commons licence and your intended use is not permitted by statutory regulation or exceeds the permitted use, you will need to obtain permission directly from the copyright holder. To view a copy of this licence, visit http://creativecommons.org/licenses/by/4.0/. 


\section{References}

1. Murakami, S.: Quantum Spin Hall Effect and Enhanced Magnetic Response by Spin-Orbit Coupling. Phys. Rev. Lett. 97, 236805 (2006). https://doi.org/10.1103/PhysRevLett.97.236805

2. Reis, F., Li, G., Dudy, L., Bauernfeind, M., Glass, S., Hanke, W., Thomale, R.: Science 357, 287 (2017). https://doi.org/10.1126/ science.aai8142

3. Wu, M., Dong, S., Yao, K., Liu, J., Zeng, X.C.: NanoLett. 16, 7309 (2016). https://doi.org/10.1021/acs.nanolett.6b04309

4. Baskaran, G.: Many-body Approaches at Different Scales: A Tribute to Norman H. March on the Occasion of his 90th Birthday. Angilella, G.G.N., Amovilli, C. (eds.) vol. 43. Cham: Springer International Publishing (2018)

5. Liao, M., Zang, Y., Guan, Z., Li, H., Gong, Y., Zhu, K., Hu, X.P., Zhang, D., Xu, Y., Wang, Y.Y., He, K., Ma, X.C., Zhang, S.C., Xue, Q.: Nat. Phys. 14, 344 (2018). https://doi.org/ 10.1038/s41567-017-0031-6

6. Xing, Y., Fu, H.L., Liu, H., Sun, Y., Wang, F., Lin, X., Wang, J., Zhang, H.M., Peng, J.P., Ma, X.C., Xue, Q.K.: Science 350, 542 (2015). https://doi.org/10.1126/science.aaa7154

7. Ilyas, A., Xiang, S., Chen, M., Khan, M.Y., Bai, H., He, P., Lu, Y., Deng, R.: Nanoscale 13, 1069 (2021). https://doi.org/10.1039/ D0NR06054B

8. Ilyas, A., Wu, H., Usman, T., Khan, S.A., Deng, R.: J. Mater. Chem. C 9, 5952-5960 (2021). https://doi.org/10.1039/D1TC00006C

9. Mannix, A.J., Zhou, X.F., Kiraly, B., Wood, J.D., Alducin, D., Myers, B.D., Liu, X., Fisher, B.L., Santiago, U., Guest, J.R., Yacaman, M.J., Ponce, A., Oganov, A.R., Hersam, M.C., Guisinger, N.P.: Synthesis of borophenes: Anisotropic, two-dimensional boron polymorphs. Science 350, 1513 (2015). https://doi.org/10.1126/science.aad1080

10. Novoselov, K.S., Geim, A.K., Morozov, S.V., Jiang, D., Katsnelson, M.I.: Nat. Phys. 2, 2006 (2006). https://doi.org/10.1038/ nphys 245

11. Grigorieva, S., Dubonos, V., Firsov, A.A.: Two-dimensional gas of massless Dirac fermions in graphene. Nature 438, 197 (2005). https://doi.org/10.1038/nature04233

12. Feng, B., Ding, Z., Meng, S., Yao, Y., He, X., Cheng, P., Chen, L., Wu, K.: Nano Lett. 12, 3507 (2012). https://doi.org/10.1021/ $\mathrm{nl} 301047 \mathrm{~g}$

13. Tongay, S., Varnoosfaderani, S.S., Appleton, B.R., Wu, J., Hebard, A.F.: Magnetic properties of MoS2: Existence of ferromagnetism. Appl. Phys. Lett. 101 (2012) 123105. https://doi. org/10.1063/1.4753797

14. Mao, X., Xu, Y., Xue, Q., Wang, W., Gao, D.: Ferromagnetism in exfoliated tungsten disulfide nanosheets. Nanoscale Res. Lett. 8, 1 (2013). https://doi.org/10.1186/1556-276X-8-430

15. Ma, Y., Dai, Y., Guo, M., Niu, C., Zhu, Y., Huang, B.: Evidence of the existence of magnetism in pristine VX 2 monolayers $(\mathrm{X}=\mathrm{S}, \mathrm{Se})$ and their strain-induced tunable magnetic properties. ACS Nano. 6 (2012) 1695. https://doi.org/10.1021/nn204667z.

16. Zhuang, H.L., Xie, Y., Kent, P.R.C., Ganesh, P.: Computational discovery of ferromagnetic semiconducting single-layer CrSnTe3. Phys. Rev. B 92, 2 (2015). https://doi.org/10.1103/PhysRevB.92. 035407

17. McGuire, M.A., Dixit, H., Cooper, V.R., Sales, B.C.: Coupling of Crystal Structure and Magnetism in the Layered, Ferromagnetic Insulator $\mathrm{CrI}_{3}$. Chem. Mater. 27, 612 (2015). https://doi.org/10. 1021/acs.chemmater.5b01781

18. Zhang, W.B., Qu, Q., Zhu, P., Lam, C.H.: Robust intrinsic ferromagnetism and half semiconductivity in stable two-dimensional single-layer chromium trihalides. J. Mater. Chem. C. 3, 12457 (2015). https://doi.org/10.1039/C5TC02840J
19. Li, X., Wu, X., Yang, J.: Half-Metallicity in $\mathrm{MnPSe}_{3}$ Exfoliated Nanosheet with Carrier Doping. J. Am. Chem. Soc. 136, 11065 (2014). https://doi.org/10.1021/ja505097m

20. Giovannetti, G., Khomyakov, P.A., Brocks, G., Karpan, V.M., van den Brink, J., Kelly, P.J.: Doping Graphene with Metal Contacts. Phys. Rev. Lett. 101, 026803 (2008). https://doi.org/10.1103/ PhysRevLett.101.026803

21. Du, K.Z., Wang, X.Z., Liu, Y., Hu, P., Utama, M.I.B., Gan, C.K., Xiong, Q., Kloc, C.: Wide-Range Band Gap, and Raman Study on Ultrathin Layers of Metal Phosphorus Trichalcogenides. ACS Nano 10, 1738 (2016). https://doi.org/10.1021/acsnano.5b05927

22. Williams, T.J., Aczel, A.A., Lumsden, M.D., Nagler, S.E., Stone, M.B., Mandrus, D.: Magnetic Correlations in the Quasi-2D Semiconducting Ferromagnet CrSiTe 3 . Phy. Rev. B 92, 144404 (2015). https://doi.org/10.1016/j.physleta.2014.10.042

23. Casto, L.D., Clune, A.J., Yokosuk, M.O., Musfeldt, J.L., Williams, T.J., Zhuang, H.L., Lin, M.W., Xiao, K., Hennig, R.G., Sales, B.C., Yan, J.Q., Mandrus, D.: Strong spin-lattice coupling in $\mathrm{CrSiTe}_{3}$. APL Mater. 3, 041515 (2015). https://doi.org/10.1063/1.4914134

24. Chittari, B.L., Park, Y., Lee, D., Han, M., MacDonald, A.H., Hwang, E., Jung, J.: Electronic and magnetic properties of singlelayer $\mathrm{MPX}_{3}$ metal phosphorous trichalcogenides. Phys. Rev. B. 94, 184428 (2016). https://doi.org/10.1103/PhysRevB.94.184428

25. Chen, X., Qi, J., Shi, D.: Strain-engineering of magnetic coupling in two-dimensional magnetic semiconductor $\mathrm{CrSiTe}_{3}$ : Competition of direct exchange interaction and superexchange interaction. Phys. Lett. A. 379, 60 (2015). https://doi.org/10.1016/j.physleta. 2016.10.017

26. Li, X., Cao, T., Niu, Q., Shi, J., Feng, J.: Coupling the valley degree of freedom to antiferromagnetic order. Proc. Natl. Acad. Sci. 110, 3738 (2013). https://doi.org/10.1073/pnas.1219420110

27. Gong, C., Li, L., Li, Z., Ji, H., Stern, A., Xia, Y., Cao, T., Bao, W., Wang, C., Wang, Y., Qiu, Z.Q., Cava, R.J., Louie, S.G., Xia, J., Zhang, X.: Discovery of intrinsic ferromagnetism in two-dimensional van der Waals crystals. Nature 546, 265 (2017). https://doi.org/10. 1038/nature22060

28. Huang, B., Clark, G., Navarro-Moratalla, E., Klein, D.R., Cheng, R., Seyler, K.L., Zhong, D., Schmidgall, E., McGuire, M.A., Cobden, D.H., Yao, W., Xiao, D., Jarillo-Herrero, P., Xu, X.: Layer-dependent ferromagnetism in a van der Waals crystal down to the monolayer limit. Nature 546, 270 (2017). https://doi.org/10.1038/nature22391

29. Velický, M., Donnelly, G.E., Hendren, W.R., McFarland, S., Scullion, D., DeBenedetti, W.J.I., Correa, G.C., Han, Y., Wain, A.J., Hines, M.A., Muller, D.A., Novoselov, K.S., Abruña, H.D., Bowman, R.M., Santos, E.J.G., Huang, F.: Mechanism of Gold-Assisted Exfoliation of Centimeter-Sized Transition-Metal Dichalcogenide Monolayers. ACS Nano 12, 10463 (2018). https:// doi.org/10.1021/acsnano.8b06101

30. Chittari, B.L., Park, Y., Lee, D., Han, M., MacDonald, A.H., Hwang, E., Jung, J.: Electronic and magnetic properties of single-layer metal phosphorous trichalcogenides. Phys. Rev. B. 94, 184428 (2016). https://doi.org/10.1103/PhysRevB.94.184428

31. Du, K.Z., Wang, X.Z., Liu, Y., Hu, P., Utama, M.I.B., Gan, C.K., Xiong, Q., Kloc, C.: Weak Van der Waals Stacking, Wide-Range Band Gap, and Raman Study on Ultrathin Layers of Metal Phosphorus Trichalcogenides. ACS Nano 10, 1738 (2016). https://doi. org/10.1021/acsnano.5b05927

32. Novoselov, K.S., Geim, A.K., Morozov, S.V., Jiang, D., Zhang, Y., Dubonos, S.V., Grigorieva, I.V., Firsov, A.A.: Electric Field Effect in Atomically Thin Carbon Films Supplementary. Science 80, 1 (2004). https://doi.org/10.1126/science.aab1343

33. Sivadas, N., Daniels, M.W., Swendsen, R.H., Okamoto, S., Xiao, D.: Magnetic ground state of semiconducting transition-metal trichalcogenide monolayers. Phys. Rev. B. 91, 235425 (2015) 
34. McGuire, M.A., Dixit, H., Cooper, V.R., Sales, B.C.: Structure and Magnetism in the Layered, Ferromagnetic Insulator $\mathrm{CrI}_{3}$. Chem. Mater. 27, 612 (2015). https://doi.org/10.1021/cm504242t

35. Deng, Y., Yu, Y., Song, Y., Zhang, J., Wang, N.Z., Sun, Z., Yi, Y., Wu, Y.Z., Wu, S., Zhu, J., Wang, J., Chen, X.H., Zhang, Y.: Gate-tunable room-temperature ferromagnetism in two-dimensional Fe3GeTe2. Nature 563, 94 (2018). https://doi.org/10.1038/s41586-018-0626-9

36. Kresse, G., Furthmüller, J.: Efficient iterative schemes for ab initio total-energy calculations using a plane-wave basis set. Phys. Rev. B. 54, 11169 (1996). https://doi.org/10.1103/PhysRevB.54.11169

37. Le Page, Y., Saxe, P.: Symmetry-general least-squares extraction of elastic data for strained materials from ab initio calculations of stress. Phys. Rev. B. 65, 104104 (2002). https://doi.org/10.1103/ PhysRevB.65.104104

38. Chabungbam, S., Sen, P.: Computational design of a robust twodimensional antiferromagnetic semiconductor. Phys. Rev. B. 96, 045404 (2017). https://doi.org/10.1103/PhysRevB.96.045404

39. Ressouche, E., Loire, M., Simonet, V., Ballou, R., Stunault, A., Wildes, A.: Magnetoelectric MnPS3 thiophosphate as a new candidate for ferrotoroidicity. Phys. Rev. B. 82, 100408 (2010). https://doi.org/10.1103/PhysRevB.82.100408

40. Rule, K.C., McIntyre, G.J., Kennedy, S.J., Hicks, T.J.: Singlecrystal and powder neutron diffraction experiments on Search for the magneti. Phys. Rev. B. 76, 134402 (2007). https://doi.org/10. 1103/PhysRevB.76.134402

41. Joy, P.A., Vasudevan, S.: Magnetism in the layered transitionmetal thiophosphates M PS 3 ( $\mathrm{M}=\mathrm{Mn}, \mathrm{Fe}$, and Ni). Phys. Rev. B. 46, 5425 (1992). https://doi.org/10.1103/PhysRevB.46.5425

42. Zener, C.: Interaction Between the Shells in the Transition Metals. Phys. Rev. 81, 440 (1951). https://doi.org/10.1103/PhysRev.81. 440
43. Kanamori, J., TerakuraJ, K.: A General Mechanism Underlying Ferromagnetism in Transition Metal Compounds. Phys. Soc. Japan. 70, 1433 (2001). https://doi.org/10.1143/JPSJ.70.1433

44. Yan, J.A., Ruan, W.Y., Chou, M.Y.: Phonon dispersions and vibrational properties of monolayer, bilayer, and trilayer graphene: Density-functional perturbation theory. Phys. Rev. B. 77, 125401 (2008). https://doi.org/10.1103/PhysRevB.77.125401

45. Michel, K.H., Verberck, B.: Theory of elastic and piezoelectric effects in two-dimensional hexagonal boron nitride. Phys. Rev. B. 80, 224301 (2009). https://doi.org/10.1103/PhysRevB.80.224301

46. Samad, A., Shafique, A., Kim, H.J., Shin, Y.H.: Superionic and electronic conductivity in monolayer W $2 \mathrm{C}$ : ab initio predictions. J. Mater. Chem. A. 5, 11094 (2017). https://doi.org/10.1039/ C7TA01177F

47. Peng, B., Zhang, H., Shao, H., Xu, Y., Ni, G., Zhang, R., Zhu, H.: Phonon transport properties of two-dimensional group-IV materials from ab initio calculations. Phys. Rev. B. 94, 245420 (2016). https://doi.org/10.1103/PhysRevB.94.245420

48. Carrete, J., Li, W., Lindsay, L., Broido, D.A., Gallego, L.J., Mingo, N.: Physically founded phonon dispersions of few-layer materials and the case of borophene. Mater. Res. Lett. 4, 204 (2016). https://doi.org/10.1080/21663831.2016.1174163

Publisher's Note Springer Nature remains neutral with regard to jurisdictional claims in published maps and institutional affiliations. 